\title{
A influência do diabetes na qualidade de vida e na imagem corporal de idosos que frequentam núcleo de convivência
}

\author{
The influence of diabetes on the quality of life and on the body image of elderly people who \\ frequent nuclei of coexistence
}

\author{
Raquel Fernandes Batista ${ }^{1}$ \\ Orcid: https://orcid.org/0000-0002-4039-5022
}

\author{
Luiz Henrique da Silva Nali ${ }^{2}$ \\ Orcid: https://orcid.org/0000-0002-8365-9796
}

\author{
Eloi Francisco Rosa ${ }^{3}$ \\ Orcid: https://orcid.org/0000-0003-1898-4573
}

\begin{abstract}
Resumo
Introdução: O diabetes é uma doença prevalente na população brasileira e é um fator complicante na qualidade de vida de idosos. Além disso, a obesidade é um dos fatores que predispõe ao diabetes, além de ser um fator apontado na percepção corporal dos idosos. Interessantemente, a percepção negativa da imagem corporal pode influenciar no desenvolvimento da obesidade. Objetivos: Identificar a influência do diabetes na qualidade de vida e na percepção da imagem corporal de idosos. Métodos: Foi realizada uma pesquisa de campo transversal com abordagem quantitativa. Os participantes responderam a três questionários sobre caracterização do perfil da amostra para avaliar a qualidade de vida e a percepção da imagem corporal. Os diabéticos responderam a um quarto questionário que avaliou a perspectiva dos pacientes sobre o impacto do diabetes e do tratamento em suas vidas. Resultados: Foram incluídos 85 idosos, 52 não diabéticos, 33 diabéticos, 29 controlados com insulina. Os resultados mostraram a qualidade de vida entre regular e boa, considerando os aspectos físicos, psíquicos, sociais e ambientais. No grupo de diabéticos destacou-se a satisfação com a capacidade de trabalhar e desempenho nas atividades de vida diária. E para a percepção da imagem corporal, o paciente não diabético relatou satisfação com sua própria imagem, enquanto os diabéticos apresentaram uma distância percebida e esperada da imagem corporal. Conclusão: A doença pode ter uma influência limitante nos aspectos físicos, emocionais e sociais, além de modificar a percepção da imagem corporal. No entanto, os idosos diabéticos mostraram-se mais satisfeitos a realizar as atividades básicas diárias.

Palavras-chave: idosos; diabetes; qualidade de vida; imagem corporal.
\end{abstract}

\begin{abstract}
Introduction: Diabetes is a prevalent disease in the Brazilian population and is a complicating factor in the quality of life of the elderly. In addition, obesity is one of the factors that predispose to diabetes and also is pointed out in the elderly body perception. Interestingly, the negative perception of the corporeal image can influence the development of obesity. Objectives: To identify the influence of diabetes on the quality of life and the perception of body image in the elderly. Methods: A crosssectional research was carried out with a quantitative approach. Participants answered three questionnaires on the characterization of the sample profile to assess quality of life and body image perception. Diabetics answered a fourth questionnaire that assessed patients' perspective on the impact of diabetes and treatment on their lives. Results: A total of 85 elderly people were included, 52 nondiabetic, 33 diabetic, 29 controlled with insulin. The results showed the quality of life between regular and good, considering the physical, psychological, social and environmental aspects. In the group of diabetics, satisfaction with the ability to work and performance in activities of daily living stood out. And for the perception of body image, the non-diabetic patient reported satisfaction with his own image, while diabetics presented a perceived and expected distance from body image. Conclusion: The disease may present a limiting influence on physical, emotional and social aspects, in addition to changing the perception of body image. However, elderly diabetics were more satisfied with performing basic daily activities.
\end{abstract}

Keywords: elderly; diabetes; quality of life; body image.

\footnotetext{
${ }^{1}$ Universidade Santo Amaro - Pós-graduação em Ciências da Saúde São Paulo, Brasil. E-mail: rfbatista@prof.unisa.br

${ }^{2}$ Universidade de Santo Amaro. E-mail: lnali@prof.unisa.br

${ }^{3}$ Universidade Santo Amaro - Pró-Reitoria. E-mail: efrosa@unisa.br
} 


\section{Introdução}

Sabe-se que a velhice é uma realidade crescente em toda a população do mundo. Esse dado é atribuído aos avanços e cuidados com a saúde e observação do contexto socioeconômico ${ }^{1}$. Por isso, a mesma não deve ser mais estigmatizada ao ser considerada apenas como declínio de funções, doenças e incapacidades ${ }^{2}$.

A população de idosos maiores de 60 anos no Brasil representa hoje 20,6 milhões de pessoas ${ }^{1}(10,8 \%$ da população total). A expectativa é que, em 2060 , o país tenha 58,4 milhões de idosos $(26,7 \%$ do total). O que explica esse aumento não é só, mas também, a melhoria da qualidade de vida, que ampliou a expectativa de vida dos brasileiros, que era de 75 anos em 2013, e passará para 81 anos em 2060 - com as mulheres vivendo, em média, 84,4 anos, e os homens 78,03 anos ${ }^{3}$. A qualidade de vida para o idoso está atrelada à manutenção e promoção da capacidade funcional, a independência, aos hábitos de vida saudáveis e à prática de atividade física ${ }^{4}$. Entretanto, os fatores psicossociais, o estilo de vida, a genética e os fatores ambientais são determinantes do processo de envelhecimento do organismo, que por sua vez sofre modificações moleculares, celulares e funcionais que podem levar à diminuição da capacidade de manutenção do equilíbrio homeostático, levando à maior disposição de doenças ${ }^{5}$.

Sabidamente, observa-se uma maior prevalência de doenças crônicas degenerativas entre os idosos. Todas as doenças cardiovasculares, respiratórias, cerebrovasculares, diabetes e até o câncer podem ser consideradas doenças crônicas. Essas representam $72 \%$ das causa mortis chamando a atenção para a hipertensão arterial sistêmica (HAS) e Diabetes Mellitus (DM). Segundo estimativas da Organização Mundial de Saúde (OMS), o número de diabéticos no mundo ultrapassava a faixa de 422 milhões em 2014, mais comum em países de média e baixa renda, no Brasil, estima-se que existam cerca de 13 milhões de pessoas vivendo com $\mathrm{DM}^{6}$. O DM é o principal responsável pelas causas de cegueira, problemas e falência renal, ataques cardíacos, acidentes vasculares cerebrais e amputações de membros inferiores. Outros dados alarmantes são os altos números de mortes associados ao diabetes de 1,6 milhão de pessoas em 2015, sendo que metade dessas mortes foi em idosos com 70 anos em média ${ }^{7}$.

O DM é uma doença crônica de etiologia múltipla, que ocorre pela deficiência do pâncreas em produzir insulina suficiente ou essa não exerce suas funções adequadamente e o organismo fica resistente a ela ${ }^{7}$. O DM se caracteriza por uma série de distúrbios metabólicos que possuem em comum, o aumento dos níveis de glicose no sangue. $O$ efeito da hiperglicemia resulta de falhas na secreção ou na função da insulina no organismo gerando complicações sistêmicas ${ }^{8,9}$.

A obesidade é considerada um dos principais fatores predisponentes do DM e HAS, são também os fatores mais apontados na percepção corporal de mulheres idosas, e menos tolerados em $60 \%$ de indivíduos idosos ${ }^{10}$. Portanto, não é difícil encontrar idosos insatisfeitos com a sua imagem corporal, que pode ser intensificada por conta de aspectos sociais relacionados à mídia e padrões de estereótipos, tais condições favorecem um rebaixamento da autoestima e da qualidade de vida ${ }^{11}$.

Por definição, a imagem corporal é a figuração mental do corpo, é o modo que o corpo se apresenta para nós. Ela surge a partir do autoconhecimento corpóreo, que envolve vivências sensoriais adquiridas no decorrer do desenvolvimento adquirido pelas vivências sensoriais que temos ao longo do processo de desenvolvimento. Isso torna-se evidente quando observamos o impacto de determinada lesão no reconhecimento postural e determinadas limitações causadas pelo trauma ${ }^{12}$. Além 
disso, é frequente observar alterações na imagem corporal em indivíduos acometidos por doenças crônicas ${ }^{12}$. Sabidamente, uma percepção negativa da imagem corporal pode influenciar no desenvolvimento de obesidade ${ }^{13}$ o que pode reverter em um fator de risco para o desenvolvimento do DM.

Portanto, é de suma importância investigar a imagem corporal de idosos diabéticos, para fins de intensificar aspectos gerais da imagem corporal em uma população idosa e identificar possíveis grupos de risco aumentado para o tratamento da doença. Sendo assim, o objetivo desse estudo foi identificar a influência do diabetes na qualidade de vida e na percepção da imagem corporal em idosos que frequentam um centro de convivência.

\section{Materiais e Métodos}

Amostra e tipo de estudo

Este estudo é um estudo transversal e aprovado pelo comitê de ética e pesquisa da Universidade Santo Amaro (61497616.5.0000.0081).

Idosos com idade igual ou superior a 60 anos e que frequentam o Núcleo de Convivência do Idoso de São Paulo (NCI) foram convidados a participar do estudo. Os voluntários foram divididos em dois grupos: grupo dos diabéticos (GDB) e grupo dos não diabéticos (GND) e foram convidados a responder alguns questionários, conforme segue.

\section{Delineamento da pesquisa}

O estudo foi conduzido no Núcleo de convivência do Idoso em São Paulo, que faz parte da sociedade beneficente Equilíbrio de Interlagos (SOBEI). A amostragem foi por conveniência.

Avaliação do nível de atividade física no qual os idosos realizaram. Dessa forma, os idosos foram classificados em ativos ou sedentários. Os idosos que realizavam mais do que 30 minutos seguidos de atividade física com frequência de cinco vezes por semana foram considerados idosos ativos14.

Em seguida os voluntários responderam o Whoqol-Bref.15 que consiste em um instrumento que avalia os seguintes domínios de qualidade de vida: Físico, Psicológico, Relações Sociais e de Meio Ambiente. Ele é composto por 26 questões de múltipla escolha, apontadas pelo sujeito de pesquisa, em uma escala de Likert, de 1 a 5, com relação à sua percepção da qualidade de vida. Além desses, os voluntários foram convidados a realizar a percepção da imagem corporal de acordo com a escala de Sörensen e Stunkard 16. As perguntas do teste consistem em uma avaliação de uma escala de percepção da imagem corporal que varia de 1 a 9 , essa escala considera de forma proporcional ao tamanho da silhueta. O teste consiste também se autoavaliar em relação à sua imagem corporal atual e como esta é esperada ou gostaria que fosse. Por fim, um último teste foi aplicado, somente para indivíduos do grupo $\mathrm{DB}$, que foi a versão brasileira da escala Problems Areas in Diabetes (B-PAID)9 Esse instrumento visa avaliar, sob a percepção dos idosos, o impacto do diabetes e do tratamento em suas vidas.

\section{Critérios de Inclusão e Exclusão}

Os critérios de inclusão foram idosos com idade maior ou igual a 60 anos, participantes ativos do NCI, não diabéticos ou diabéticos, com a glicemia controlada, segundo informação dos mesmos e comprovada por documentos entregues, como: receituários das medicações; hemograma ou pela caderneta da pessoa idosa. Além de não possuírem morbidades incapacitantes físicas ou mentais que impedissem a compreensão das perguntas e comandos dos instrumentos de avaliação.

Foram excluídos desta pesquisa idosos que não frequentassem o NCI, que não tivessem idade igual ou maior a 60 anos, que mencionassem glicemia descontrolada mesmo com uso de 
medicação, ou que possuíssem comorbidades incapacitantes ou rebaixamento cognitivo que os impedissem de preencher os instrumentos propostos. Além disso, indivíduos que se recusassem assinar o TCLE.

\section{Procedimentos}

Após a aplicação dos questionários os dados foram tabulados e as variáveis obtidas foram utilizadas para realização da análise estatística, sendo que foi utilizado o anova de três fatores, variáveis com $p<0,05$ entre as comparações foram consideradas significativas.

\section{Resultados}

O estudo contou com a participação de 85 idosos diabéticos e não diabéticos, 09 homens e 76 mulheres, que frequentavam as atividades do NCI semanalmente, e que praticavam ou não, atividade física. Dos 85 idosos entrevistados, 52 deles (61\%) fizeram parte do grupo de não diabéticos (GND), enquanto 33 (38\%) do grupo de diabéticos (GDB), sendo que 29 desses faziam uso da insulina. A condição da doença foi autorreferida, porém comprovada através de documentos (receituários das medicações de controle glicêmico, bem como a caderneta da pessoa idosa que possui o controle glicêmico, inclusive níveis de glicemia em exames laboratoriais em posse dos idosos.

Tanto no GND quanto no GDB foram observadas diversas comorbidades, que podem ser observadas na tabela 1 .

Tabela 1. Principais comorbidades dos pacientes do GDB e GND. Legenda: GDB Grupo diabetes; GND Grupo sem diabetes

\begin{tabular}{lcc}
\hline \multicolumn{1}{c}{ Comorbidades } & GDB(\%) & GND(\%) \\
\hline Hipertensão Arterial Sistêmica & $25(29,8)$ & $33(29,2)$ \\
Cardiopatias & $5(6)$ & $12(10,6)$ \\
Catarata & $15(17,9)$ & $12(10,6)$ \\
Artrose & $19(22,6)$ & $30(26,5)$ \\
Osteoporose & $8(9,5)$ & $14(12,4)$ \\
Problemas na tireoide & $10(11,9)$ & $7(6,2)$ \\
Fibromialgia & $2(2,4)$ & $5(4,4)$ \\
\hline Total & $\mathbf{8 4}$ & $\mathbf{1 1 3}$ \\
\hline
\end{tabular}

Dentre todos os entrevistados 82,5\% referiram praticar alguma atividade física em média de 4 a 5 vezes por semana, durante 30 minutos ou mais. Dentre o GND, 90,5\% faziam atividade física cerca de cinco vezes por semana. Enquanto o GDB $69,6 \%$ realizavam, 4 vezes por semana, 30 minutos ou mais.

Em relação à qualidade de vida $(\mathrm{QV})$, os dados foram analisados pelo Whoqol-Bref, observou-se que nos dois grupos a qualidade de vida foi classificada com médias entre 3 e 4 , consideradas pelo instrumento qualidade regular e boa, em praticamente todos os domínios, conforme tabela 2. Observou-se diferença estatística nos domínios 17 e 18 do domínio físico, esses compreendem a capacidade de desempenho nas Atividades de vida diárias (AVD) e satisfação capacidade para o trabalho. Os indivíduos do GDB apresentaram-se mais satisfeitos para a capacidade no desempenho das AVD e para a capacidade de trabalho do que os indivíduos do GND. 
Tabela 2. Pontuação dos principais domínios de pacientes do GDB e GND de acordo com o teste do WHOQOL-Bref Legenda: MF= Média Final GDB $=$ Grupo diabetes; $G N D=$ Grupo sem diabetes.

\begin{tabular}{|c|c|c|}
\hline & $\underline{\text { GDB média }}$ & GND média \\
\hline 1Autoavaliação QV & $4 \pm 0,1$ & $4 \pm 0,1$ \\
\hline 2Satisfação Saúde & $3,5 \pm 0,1$ & $4 \pm 0,1$ \\
\hline \multicolumn{3}{|l|}{ Domínio Físico } \\
\hline 3Impedimento por Dor & $4 \pm 0,2$ & $4 \pm 0,1$ \\
\hline 4T to médico para levar a vida & $3 \pm 0,1$ & $3 \pm 0,1$ \\
\hline 10 Energia suf. Dia a dia & $4 \pm 0,1$ & $4 \pm 0,1$ \\
\hline 15 capacidade locomover & $4 \pm 0,1$ & $4 \pm 0,1$ \\
\hline 16 satisfação sono & $3,4 \pm 0,2$ & $3,5 \pm 0,1$ \\
\hline 17 Satisf. cap. desemp. AVD & $4,17 \pm 0,02 *$ & $3,82 \pm 0,02$ \\
\hline 18 Satisf. cap. trabalho & $4,6 \pm 0,0001 *$ & $3,82 \pm 0,0001$ \\
\hline MF & $3,89 \pm 0,04$ & $3,71 \pm 0,04$ \\
\hline \multicolumn{3}{|l|}{ Domínio Psíquico } \\
\hline 5 Quanto aproveita vida & $3,8 \pm 0,4$ & $3,7 \pm 0,4$ \\
\hline 6 Quanto a vida tem sentido & $4,2 \pm 0,8$ & $4,1 \pm 0,8$ \\
\hline 7 Concentração & $3,13 \pm 0,7$ & $3,21 \pm 0,7$ \\
\hline 11 Aceitação aparência física & $4 \pm 0,2$ & $4 \pm 0,1$ \\
\hline 19 Satisfação consigo mesmo & $4,2 \pm 0,1$ & $4,1 \pm 0,09$ \\
\hline 26 Freq sentimentos neg & $4 \pm 0,2$ & $3,6 \pm 0,1$ \\
\hline $\mathrm{MF}$ & $4 \pm 0,1$ & $4 \pm 0,1$ \\
\hline \multicolumn{3}{|l|}{ Domínio Social } \\
\hline 20 satisf. relaç. pessoais & $4,5 \pm 0,1$ & $4 \pm 0,1$ \\
\hline 21 sat. vida sexual & $4 \pm 0,1$ & $4 \pm 0,1$ \\
\hline 22 Apoio dos amigos & $4 \pm 0,1$ & $4 \pm 0,1$ \\
\hline MF & $4 \pm 0,1$ & $4 \pm 0,1$ \\
\hline \multicolumn{3}{|l|}{ Domínio meio Ambiente } \\
\hline 8 segurança vida diária & $4 \pm 0,1$ & $3,6 \pm 0,1$ \\
\hline 9 ambiente saudável & $3,6 \pm 0,1$ & $3,5 \pm 0,1$ \\
\hline $12 \$$ suf. satisfazer necessidade & $3 \pm 0,1$ & $2,94 \pm 0,1$ \\
\hline 13Disponib/e infos & $3,27 \pm 0,2$ & $3,28 \pm 0,1$ \\
\hline 14 Oportunidade de ativ. lazer & $3,5 \pm 0,2$ & $3,38 \pm 0,1$ \\
\hline 23Satisf. local mora & $4,2 \pm 0,1$ & $4,0 \pm 0,1$ \\
\hline 24 Satisf. serviços saúde & $3,13 \pm 0,2$ & $2,86 \pm 0,1$ \\
\hline 25 Satisf. meio de transporte & $3,51 \pm 0,1$ & $3,4 \pm 0,1$ \\
\hline MF & $3,53 \pm 0,07$ & $3,39 \pm 0,07$ \\
\hline
\end{tabular}

$\mathrm{Na}$ análise dos dados do B-PAID realizado no GDB destacaram-se problemas relacionados à condição emocional dos idosos, como por exemplo: preocupação em lidar com possíveis complicações da doença (escore de 62,5); sentimento de culpa com a própria negligência na aderência ao tratamento (escore de 45,28). Além disso, os idosos também relataram outras condições como falta de metas claras e concretas no cuidado do diabetes (escore de 61,2), privação a respeito de comida e refeições (escore de 46,25) e também falta de apoio de amigos e familiares no tratamento da doença (escore de 27,5). $\mathrm{Na}$ análise da escala de silhueta foi 
verificada a presença da percepção corporal de acordo com uma escala que variou de 1 a 9 , em que 1 seria extremamente magro enquanto o 9 seria extremamente obeso. As frequências das notas dos grupos de silhuetas estão descritas na tabela 3 .

Tabela 3. Frequência na percepção da imagem corporal real e esperada nos GDB

\begin{tabular}{|c|c|c|c|c|}
\hline \multirow{3}{*}{$\begin{array}{l}\text { Grupos das } \\
\text { silhuetas }\end{array}$} & \multicolumn{2}{|c|}{ GDB } & \multicolumn{2}{|c|}{ GND } \\
\hline & Imagem & Imagem & Imagem & Imagem \\
\hline & Corporal Real & Corporal & Corporal Real & Corporal \\
\hline & $(\%)$ & Esperada (\%) & $(\%)$ & Esperada (\%) \\
\hline 1 e 2 & $6(20,7)$ & $12(41,4)$ & $4(7,8)$ & $12(23,5)$ \\
\hline 3 & $2(6,9)$ & $8(27,6)$ & $6(11,8)$ & $17(33,3)$ \\
\hline 4 e 5 & $15(51,7)$ & $8(27,6)$ & $26(51,0)$ & $17(33,3)$ \\
\hline 6 e 7 & $6(20,7)$ & $1(3,4)$ & $14(27,5)$ & $5(9,8)$ \\
\hline 8 e 9 & - & - & 1 & - \\
\hline
\end{tabular}

\section{Discussão}

No nosso grupo amostral foi observado um predomínio de mulheres em comparação aos homens, ( $\mathrm{n}=76$ mulheres para $\mathrm{n}=09$ homens), com média de idade de $71 \pm 5$ anos. Mulheres com 75 anos ou mais, representam mais da metade da população dessa faixa etária de países como Brasil e África do Sul 17,18. A feminização do envelhecimento tem sido uma preocupação mundial, pois mulheres idosas estão cada vez mais expostas aos problemas inerentes às desigualdades sociais, financeiros e às limitações de saúde em fases mais avançadas 17,18.

Dentre os fatores determinantes de morbimortalidade estão o DM e HAS que são as doenças crônicas mais frequentes em idosos com considerável incidência em todo o mundo. Em razão desses fatores, atrela-se a necessidade de aceitação por parte do idoso às mudanças de hábitos rotineiros que são fundamentais e, em geral, devem ser estabelecidas continuamente19. Além disso, sabe-se que os diabéticos apresentam um risco maior de desenvolvimento de HAS em relação a indivíduos saudáveis.

Nosso estudo apontou prevalência semelhante de HAS entre os grupos estudados, esse dado, em teoria, não era esperado, uma vez que a frequência de HAS geralmente é maior entre indivíduos diabéticos, quando comparados a indivíduos saudáveis. No entanto, no GND, a ocorrência de outros fatores que possam contribuir para a frequência de HAS também estava presente. Vale destacar que existem outras comorbidades também associadas ao diabetes, como as dislipidemias, sobrepeso, obesidade e sedentarismo 17,20. Um estudo anterior descreveu a elevada frequência de hipertensão, diabetes e artrite/artrose em idosos 21, achados que corroboram os nossos dados.

Apesar de a catarata estar presente em idosos diabéticos com tempo de diagnóstico geralmente maior de 20 anos, esse dado merece atenção pelo fato de aumentar a necessidade de suporte nas AVD, afetando diretamente na autonomia, funcionalidade e qualidade de vida do idoso20.

Missias et al.4 ressalta que uma velhice com boa qualidade de vida é considerada saudável quando está relacionada com a afetividade e a prática de atividades físicas. Grillo20 aponta a necessidade de mudança nos hábitos de vida associando a pratica de atividade física, alimentação saudável, qualidade do sono, 
independência funcional e social, reflete em benefícios modestos, mas significativos em longo prazo na função física, principalmente em adultos de meia idade e idosos obesos com diabetes tipo 2 .

Pilger, et al. 21 afirmam não perceberem diferença na qualidade de vida, entre os participantes diabéticos e não diabéticos que praticavam ou não atividade física. Os apontamentos dos autores contribuem com esses achados que também não se verificou diferença estatística na frequência das atividades físicas entre os idosos DB e ND avaliados, e também na qualidade de vida.

O Whoqol-bref, instrumento que permite a avaliação da qualidade de vida de forma geral, evidenciou que os idosos estavam satisfeitos com a qualidade de vida e a saúde deles. E apesar de não ter apresentado significância estatística, nos diferentes domínios, cabe destacar que, por se tratar de pessoas idosas, a média tende a diminuir de acordo com as dificuldades do próprio processo de envelhecimento $\mathrm{e}$ torna-se um dado importante que deve ser mantido ou até melhorado.

Sabidamente o diabetes pode influenciar em aspectos de trabalho $\mathrm{e}$ emocionais 22. No entanto, foi observado que os idosos do GDB apresentaram uma maior satisfação e capacidade para o desenvolvimento das AVD e também associadas ao trabalho. Uma possível explicação para esse achado, é pelo fato dos idosos relacionarem a sua condição atual com uma possível capacidade no desenvolvimento de atividades básica. Um estudo anterior descreveu que a maioria dos idosos diabéticos associava o grau de satisfação, sobretudo ao seu bem-estar físico 2. Portanto, acredita-se que a satisfação no desenvolvimento de AVD está ligada à independência desses idosos 23 .

$\mathrm{Na}$ escala B-PAID, foi observada uma tendência na preocupação com as complicações associadas ao diabetes e o quanto os idosos se sentiam culpados quando deixavam de tomar os cuidados necessários para controle da doença. Quanto aos problemas relacionados ao tratamento, referiram sentir a falta de metas claras e concretas no cuidado do diabetes. Apesar da falta, existe a necessidade de reforçar para os idosos e seus familiares constantemente, referentes aos cuidados de monitorar a glicemia, autoaplicar as injeções de insulina e usar corretamente as medicações. Esses cuidados ajudam a elevar a adesão aos tratamentos e dietas e estimulam a pratica de atividades física 9 .

O teste de silhuetas evidenciou uma percepção corporal discrepante, principalmente no GDB, que evidenciou o desejo de uma silhueta bem menor do que apontavam ter. Apesar de registrar uma percepção individual, sabe-se que a aceitação da própria imagem, fortalece a autoestima, interfere na qualidade vida e nas motivações pessoais. A imagem do corpo é o eixo principal da personalidade e representa o equilíbrio entre a identidade do idoso e sua relação no contexto entre corpo e o meio 24 .

Quanto mais distante for a imagem corporal percebida da esperada, mais prejuízos corporais, emocionais e sociais, que podem interferir no autocuidado. Cavalcante 25 afirma que a identidade é construída a partir do corpo e que a identidade do idoso origina-se no corpo simbólico, que encontra suporte no corpo real. Conforme o corpo sofre interferências e modificações, acontecem progressivos comprometimentos da identidade, com riscos de conflitos e dificuldades, principalmente quando a personalidade é desenvolvida sobre a imagem real do seu corpo. $\mathrm{O}$ fato dos idosos realizarem os trabalhos em grupo de forma regular, ao longo do tempo, tornam eficazes os resultados para o controle da glicemia, do conhecimento do diabetes e de alguns comportamentos relacionados à diabetes 17 . 


\section{Conclusão}

De acordo com os nossos dados conclui-se que o diabetes aparentemente influencia na percepção da imagem corporal. No entanto, nas questões de qualidade de vida, os idosos com diabetes apresentaram-se mais satisfeitos com a sua capacidade de realização das AVD.

\section{Referências}

1. Veras R. Envelhecimento populacional contemporâneo: demandas, desafios e inovações. Rev Saude Publica. 2009 Jun;43(3):548-54.

2. Dawalibi NW, Anacleto GMC, Witter C, Goulart RMM, Aquino R de C de. Envelhecimento e qualidade de vida: análise da produção científica da SciELO. Estud Psicol. 2013 Sep;30(3):393-403.

3. Ministério da Saúde. Brasil é reconhecido por políticas públicas em favor de idosos — Legado Brasil [Internet]. [cited 2019 Sep 15]. Available from: http://legado.brasil.gov.br/noticias/saude/2014/01/brasil-e-reconhecido-por-politicas-publicasem-favor-de-idosos/agenciabrasil190812mca_4.jpg/view

4. Moreira RM, Santos CES dos, Couto ES, Teixeira JRB, Souza RMMM. Qualidade de vida, Saúde e Política Pública de Idosos no Brasil: uma reflexão teórica. Vol. 16, Revista Kairós : Gerontologia. 2009. 27-38 p.

5. Leme L. A Gerontologia e o problema do envelhecimento. Visao historica. Gerontol a velhice e o Envelhec em visao Glob. 2002;

6. Bertoldi AD, Kanavos P, França GVA, Carraro A, Tejada CA, Hallal PC, et al. Epidemiology, management, complications and costs associated with type 2 diabetes in Brazil: a comprehensive literature review. Global Health. 2013 Dec 3;9(1):62.

7. World Health Organization. Diabetes [Internet]. 2018 [cited 2019 Sep 15]. Available from: https://www.who.int/news-room/fact-sheets/detail/diabetes

8. Duarte E, Marques A, Leal M, Melo GP, Silva C. Idosos diabéticos Autopercepção do estado geral de saúde. CIAIQ2015. 2015 Jul 16;1.

9. Gross CC. Versão brasileira da escala PAID(Problem Areas in Diabetes) : avaliação do impacto do diabetes na qualidade de vida. 2004;

10. Knight T, Illingworth K, Ricciardelli L. Health Implications of Body Size Perception and Weight Tolerance in Older Adults. J Health Psychol. 2009 Apr;14(3):425-34.

11. Ferreira AA, Menezes MFG, Tavares EL, Nunes NC, Souza FP de, Albuquerque NAF, et al. Estado nutricional e autopercepção da imagem corporal de idosas de uma Universidade Aberta da Terceira Idade. Rev Bras Geriatr e Gerontol. 2014;17(2):289-301.

12. Scatolin HG. A imagem do corpo: as energias construtivas da psique. Psicol Rev. 2012 Feb 6;21(1):115-20.

13. Bjerggaard M, Philipsen A, Jørgensen ME, Charles M, Witte DR, Sandbæk A, et al. Association of self-perceived body image with body mass index and type 2 diabetes-The ADDITION-PRO study. Prev Med (Baltim). 2015 Jun;75:64-9.

14. WHO | Physical Activity and Older Adults. WHO. 2015; 
15. Fleck MP, Louzada S, Xavier M, Chachamovich E, Vieira G, Santos L, et al. Aplicação da versão em português do instrumento abreviado de avaliação da qualidade de vida \&quot;WHOQOL-bref\&quot; Rev Saude Publica. 2000 Apr;34(2):178-83.

16. Débora Cristiane Machado, Nara Sudo AHGP. Body image of elderly living in long-stay residences for the aged in Porto Alegre-RS. Vol. 5, CERES: Nutrição \& Saúde. 2010. 139-148 p.

17. Menon K, Mousa A, Courten MP de, Soldatos G, Egger G, Courten B de. Shared Medical Appointments May Be Effective for Improving Clinical and Behavioral Outcomes in Type 2 Diabetes: A Narrative Review. Front Endocrinol (Lausanne). 2017;8:263.

18. Prefeitura de São Paulo. Informes Urbanos: Cresce número de idosos na cidade de São Paulo. Inf Urbanos. 2011;3:1-3.

19. Marianne Pinheiro da Rocha, Renata Furlan Viebig, Andrea Romero Latterza. Imagem corporal em idosos: influências dos hábitos alimentares e da prática de atividade física [Internet]. EFDeportes. 2012 [cited 2019 Sep 18]. Available from:

https://www.efdeportes.com/efd166/imagem-corporal-em-idosos-influencias.htm

20. Grillo M de FF, Gorini MIPC. Caracterização de pessoas com Diabetes Mellitus Tipo 2. Rev Bras Enferm. 2007 Feb;60(1):49-54.

21. Pilger C, Menon MH, Mathias TA de F. Socio-demographic and health characteristics of elderly individuals: support for health services. Rev Lat Am Enfermagem. 2011 Oct;19(5):1230-8.

22. Krstović-Spremo V, Račić M, Joksimović BN, Joksimović VR. The effects of diabetes mellitus and hypertension on work productivity. Acta Med Acad. 2014 Nov 15;43(2):122-33.

23. Franchi KMB, Monteiro LZ, Almeida SB de, Pinheiro MHNP, Medeiros AIA, Montenegro RM, et al. CAPACIDADE FUNCIONAL E ATIVIDADE FISICA DE IDOSOS COM DIABETES TIPO 2. Rev Bras Atividade Física Saúde. 2008;13(3):158-66.

24. Neto F. Manual da Avaliação Motora para a Terceira Idade. Porto Alegre; 2009. 268 p.

25. Cavalcante A. M. Psiquiatria, outros olhares A psicologia do idoso [Internet]. Psychiatry online Brasil. 2002 [cited 2019 Sep 15]. p. 5. Available from:

http://www.polbr.med.br/ano02/mour0502.php

\section{Como citar este artigo:}

Batista RF, Nali LHS, Rosa EF. A influência do diabetes na qualidade de vida e na imagem corporal de idosos que frequentam núcleo de convivência. Rev. Aten. Saúde. 2021; 19(69): 715. 